\title{
Insights into Pore Size Control in Cellulose Nanopapers Through Modeling and Experiments
}

\author{
G. P. Szekeres ${ }^{1,2}$, Z. Nemeth ${ }^{1,2}$, K. Schrantz ${ }^{1,3}$, K. Hernadi' ${ }^{2}$, and T. Graule ${ }^{1}$ \\ ${ }^{1}$ Empa, Swiss Federal Laboratories for Materials Science and Technology, Laboratory for High Performance Ceramics, \\ Überlandstrasse 129, Dübendorf, $\mathrm{CH}-8600$, Switzerland \\ ${ }^{2}$ Department of Applied and Environmental Chemistry, University of Szeged, Rerrich tér 1, Szeged H-6720, Hungary \\ ${ }^{3}$ Department of Inorganic and Analytical Chemistry, University of Szeged, Dóm tér 7, Szeged H-6720, Hungary
}

\begin{abstract}
An easy way of controlling pore sizes during the preparation of cellulose nanopapers using nanofibrillated cellulose and different solvents, such as water, ethanol and acetone, was applied in this study. A possible mathematical model is also presented, that describes the occuring processes, which model is based on simple probability theory computations taking the number of possible hydrogen bonds into consideration. This model allows the better understanding of the solvent dependence of pore formation on a molecular level. For the comparison of the effects of solvents two different series of cellulose nanopapers were prepared. In the cases of both series, an aqueous nanofibrillated cellulose suspension was used for the fabrication of nanopapers, and different solvents were used for their modification. Based on scanning electron microscopy images and mercury intrusion porosimetry data it has been concluded, that using different solvents was a crucial point in controlling pore sizes. A theory about the swelling effects, as well as the formation and decomposition of nanofibrillated cellulose aggregates based on the hydrogen bonding abilities of the solvents, was proposed and proven in this paper. As-prepared nanocellulose papers can be excellent candidates for further applications as support materials (e.g., virus filtration).
\end{abstract}

Keywords: Nanocellulose, Controlled Porosity, Ethanol, Acetone, Virus Filtration.

\section{INTRODUCTION}

Cellulose is the most abundant organic material on Earth. ${ }^{1}$ Due to its long, linear chains built up by $\beta-1,4$ glycoside linked anhydroglucose monomers it is the subject of both organic and polymer chemistry. Over 1000 tons of cellulose is isolated every year, ${ }^{2}$ mostly from wood pulp, but other sources, i.e., hemp, ${ }^{3}$ bamboo, ${ }^{4}$ cotton, ${ }^{5}$ etc. are also significant. Regarding its nano-sized fabrication, two forms of nanocellulose are considered to be the most significant: nanocrystalline cellulose (nanowhiskers) ${ }^{6}$ and nanofibrillated cellulose (NFC). ${ }^{7}$ In the latter case, cellulose chains form elementary fibrils which, attaching to each other, construct a cellulose nanofibril. Nanofibrillated cellulose has a paracrystalline structure, which means that crystalline and amorphous segments are alternating along the fibrils. Its morphology provides the NFC flexibility and high relative surface area as well. ${ }^{8}$ As NFC is biodegradable and does not show any signs of cytotoxicity these properties

\footnotetext{
${ }^{*}$ Author to whom correspondence should be addressed.
}

combined with its high length-to-width aspect ratio-with tens of nanometers width and several microns lengthmake NFC an optimal polymer filler material, especially for biodegradable polymers. ${ }^{9-11}$

In the last couple of decades several possible NFC applications have been reported, including its use in tissue engineering, electroacoustic membranes, clothing, packing materials, purification techniques, gas barriers, etc. ${ }^{11-13}$ In most cases NFC fibers are in the form of hydrogel, aerogel, foam or nanopaper. ${ }^{12}$ Cellulose nanopaper is a compact material which is obtained after the filtration of a NFC suspension. ${ }^{14,15}$ However, the filtration and drying of an aqueous NFC suspension results in a film with very low porosity. ${ }^{16}$ There are several techniques for enhancing the porosity, e.g., solvent exchange, supercritical drying, etc. Supercritical drying results in the highest porosity according to Sehaqui et al., ${ }^{16}$ but solvent exchange is a much more comfortable way to increase this property in order to make cellulose nanopapers applicable in adsorption utilizations such as gas barriers. ${ }^{11}$ 
Despite the successful investigations on porosity and pore size control, the understanding of the ongoing mechanisms during the preparation of cellulose nanopapers is still a very challenging topic. In connection with the above cited reports focusing on the effects of different solvents on the cellulose nanopaper structure, our study discusses the changes in pore size distribution when acetone, ethanol or their $50-50 \mathrm{~V} / \mathrm{V} \%$ mixture was used as exchanging solvent. The morphology of the as-prepared cellulose nanopapers was characterized by scanning electron microscopy (SEM) and porosity data were obtained from mercury intrusion porosimetry (MIP). A theory based on the swelling properties of cellulose and the hydrogen bonding affinity of the used solvents is proposed and proven with a simplified mathematical model. With this simplified mathematical model the supposedly most effective interactions are successfully represented in the system subject to our observations. Efficient and cheap nanocellulose filter bases of different permeability can be fabricated by this facile technique. One of our main goals in the near future is to develop innovative nanocomposite based filters, and to investigate their surface properties and adsorption capability in order to improve drinking water quality by removing viruses from contaminated water.

\section{EXPERIMENTAL DETAILS}

Elemental Chlorine Free fibers (ECF) from bleached softwood-based pulp-fibers (Picea abies and Pinus spp.) were purchased from Stendal (Berlin, Germany). Acetone and ethanol were obtained from Thommen Furler AG (Büren, Switzerland) and from Alko Swiss $\mathrm{GmbH}$ (Wildegg, Switzerland), respectively. Deionized waterproduced in the laboratory by osmotic purification-was used for the experiments and rinsing.

To prepare the $\sim 1.5 \mathrm{wt} \% \quad \mathrm{NFC}$ suspension, $2 \%$ ECF aqueous suspension was processed by a "Supermass Colloider" (MKZA10-20J CE) equipped with non-porous grinding stones rotating at the nominal velocity (ca. $1500 \mathrm{rpm}$, motor-load: $15 \mathrm{~kW}$ ). The instrument was purchased from Masuko Sangyo Co., Ltd. (Kawaguchi/Saitama, Japan).

Cellulose nanopapers were prepared by the 'papermaking' method as follows, similar to that proposed by Sehaqui et al. ${ }^{16} 6.67 \mathrm{~g}$ of NFC suspension (containing about $100 \mathrm{mg}$ NFC) was diluted to $30-40 \mathrm{~mL}$ by the selected solvent to make it less viscous. The diluted suspension was filtered through a Durapore ${ }^{\circledR}$ PVDF membrane with the uniform pore size of $0.1 \mu \mathrm{m}$. The filtration process was enhanced by a Divac $1.2 \mathrm{~L}$ vacuum pump. After eliminating as much solvent as possible the membrane with the filter cake was dried on air at room temperature. The resulting white, non-transparent, flexible cellulose nanopaper spontaneously detached from the filter membrane. The control sample, which was dried at $100{ }^{\circ} \mathrm{C}$
Table I. The prepared cellulose nanopapers and their codes.

\begin{tabular}{|c|c|c|}
\hline Sample code & Suspending agent & Washing agent \\
\hline Control & Water & Water \\
\hline Cell $^{\text {ac }}{ }_{S} \mathrm{~S}$ & Acetone & Acetone \\
\hline $\mathrm{Cell}^{\mathrm{et}} \_\mathrm{S}$ & Ethanol & Ethanol \\
\hline Cell ${ }^{\text {ac-et }} \_S$ & $\begin{array}{l}50-50 \mathrm{~V} / \mathrm{V} \% \\
\text { acetone-ethanol }\end{array}$ & $\begin{array}{l}50-50 \mathrm{~V} / \mathrm{V} \% \\
\text { acetone-ethanol }\end{array}$ \\
\hline Cell $^{\mathrm{ac}} \_\mathrm{W}$ & Water & Acetone \\
\hline Cell ${ }^{\mathrm{et}} \_\mathrm{W}$ & Water & Ethanol \\
\hline Cell ${ }^{\text {ac-et }} \_W$ & Water & $\begin{array}{l}50-50 \mathrm{~V} / \mathrm{V} \% \\
\text { acetone-ethanol }\end{array}$ \\
\hline
\end{tabular}

in a drying furnace, turned out to be a flexible, transparent, plastic-like film. The increased temperature had no influence on the structure and consistence of the prepared film; it was only applied for the acceleration of the drying process.

Two series of sample preparation were carried out. In the first series the NFC suspension was diluted by acetone, ethanol or their $50-50 \mathrm{~V} / \mathrm{V} \%$ mixture and after filtration the filter cake was rinsed with the same solvent (later referred to as S-series deriving from "suspended in the marked solvent"). In the second series the NFC suspension was diluted with water and after filtration the filter cake was washed with the selected solvent (referred to as W-series from "washed with the marked solvent"). The prepared samples and their codes are listed in Table I.

The morphology of the as-prepared nanopapers was investigated by scanning electron microscopy (SEM). The samples were first attached to a conductive carbon tape, and then sputtered with a 5-nm-thick platinum layer. A FEI Nova NanoSEM 230 microscope, which operated in the $10-15 \mathrm{kV}$ range, was used to obtain visual data of surface structures.

As porosity properties play an important role in cellulose nanopaper applications mercury intrusion porosimetry (MIP) was also carried out. These measurements consisted of two separate parts: low pressure porosimetry (up to $395 \mathrm{kPa}$ ) data were provided by a PASCAL 140 porosimeter, while the high pressure investigations (up to $200 \mathrm{MPa}$ ) took place in a PASCAL 440 porosimeter. The obtained data were then combined and extracted as histograms by S.O.L.I.D. software. All presented data are the average of 4-8 parallel measurements.

\section{RESULTS AND DISCUSSION}

Since the surface of the nanopapers of the S-series looked rougher even for the naked eye than that of the $\mathrm{W}$-series, microscopic investigations were done for further confirmation of conclusions. In the SEM images it can be seen that the surface of the control sample is coherent (Figs. 1( $a$ and b)), while there are different sized holes on the surface of the other samples (Figs. 1(c and e)). In case of the S-series (represented by Cell ${ }^{\text {ac }}{ }_{-}$S in Fig. 1(c)) the 

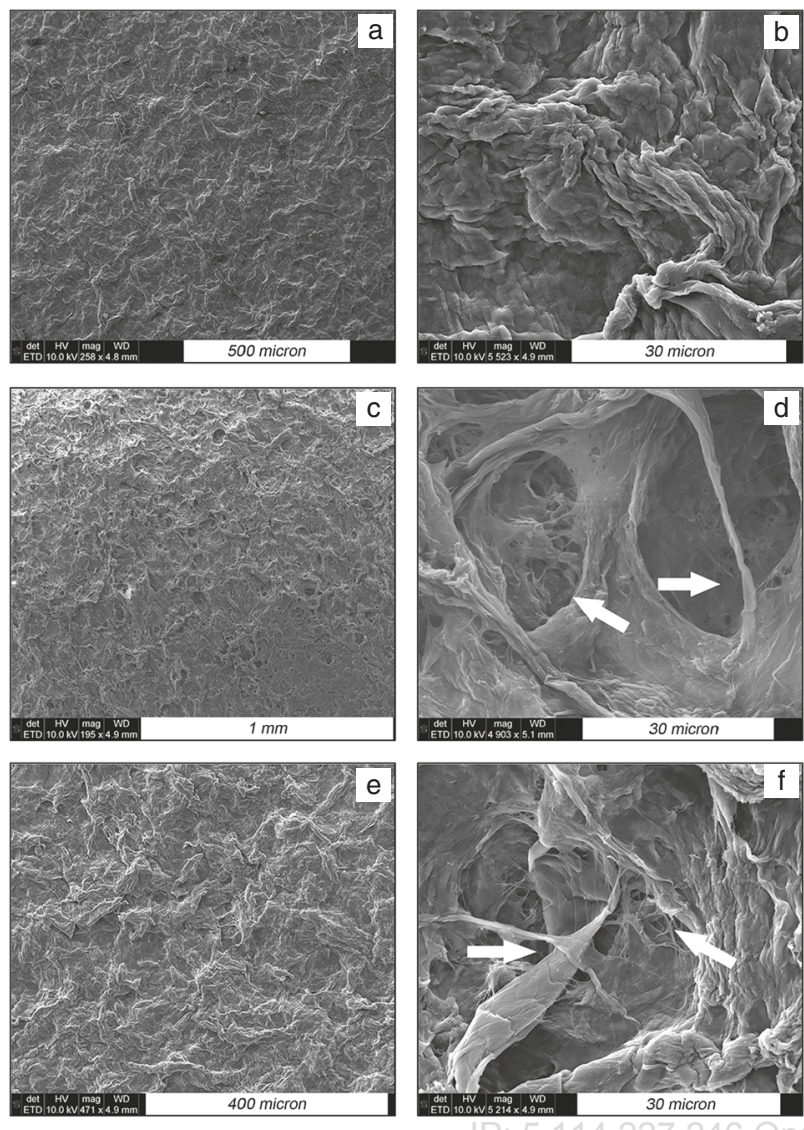

Figure 1. SEM images of the control sample (a, b) and selected samples Cell ${ }^{\text {ac }} \_$S $(c, d)$ and Cell ${ }^{\text {et }} \_$W $(e, f)$ in two different magnifications. The control sample has a coherent surface compared to Cell ${ }^{\text {ac }}$ S $S$ and Cell ${ }^{\text {et }}$ W where holes resulting from aggregate stacking (marked with white arrows) can be seen.

holes at the surface are already observable at lower magnifications. The upper layer of the $\mathrm{W}$-series samples was smoother and the prevalence of holes was smaller than in the samples of the S-series. It can also be concluded that the samples prepared with acetone always had a rougher surface than those prepared with ethanol.

The holes on the surface obviously do not correlate directly with porosity but they represent the intensity of aggregate formation in different solvents, which eventually leads to enhanced porosity. In images with higher magnification (Figs. 1(d and f)) it is visible how aggregates are formed and stacked on each other (marked by white arrows). As cellulose has a decreasing swelling property in the row of water, ethanol and acetone ${ }^{17}$ it is proposed that the prevalence of holes and therefore the growing amount of aggregated NFC leads to bigger pores. To prove this assumption, MIP measurements were carried out.

After the combination of low pressure and high pressure porosimetric data cumulative and relative pore volume versus pore size histograms were obtained. The pore size distribution of the S-series are presented in Figure 2.

The control sample showed a very low porosity of $4.5 \%$. The size of its pores altered in the $10-100 \mathrm{~nm}$ interval.
As it is shown in Figure 2, the nanopapers of S-series had a pore size distribution in the micrometer range. The highest column in the histograms of Cell ${ }^{\text {ac }} \_$S and Cellet ${ }_{-} \mathrm{S}$ were those with the medium of $3802 \mathrm{~nm}$ and $2630 \mathrm{~nm}$, respectively (marked with arrows in Fig. 2). This difference might be the result of different interaction strengths between the suspending agents and NFC. Cellulose nanofibers can order in stable aggregates due to the high number of hydrogen bonds between the fibers. ${ }^{18}$ When a solvent in which cellulose has better swelling properties is used as suspending agent aggregates are less likely formed because cellulose fibers interact with the solvent molecules with higher affinity than with another fiber. However, when a suspending agent is used, in which NFC has worse swelling properties, NFC favors making aggregates instead. As acetone is an aprotic solvent only acting as acceptor in hydrogen bonds (Table II), bigger aggregates tend to be formed when it is used as suspending agent. When these aggregates subside, in contrast to the sedimentation stand-alone fibers, bigger pores are formed due to worse ordering properties. Ethanol, in which NFC has better swelling properties than in acetone, does not force NFC to form aggregates as intensively, thus smaller pores become more abundant.

The two pore size ranges (the width of the histographic column on the $x$-axis) for sample Cell ${ }^{\text {ac-et }}{ }_{-} \mathrm{S}$ with the biggest relative numerical pore volume values $\left(\mathrm{mm}^{3} / \mathrm{g}\right)$ have the medium of $3802 \mathrm{~nm}$ and $2630 \mathrm{~nm}$, respectively (Fig. 2). These values correspond to the values measured separately for samples $\mathrm{Cell}^{\mathrm{ac}}{ }_{-} \mathrm{S}$ and $\mathrm{Cell}{ }^{\mathrm{et}} \_\mathrm{S}$, suggesting that the solvent molecules, when present in a mixture, show the same influence on the characteristic pore size as they did separately. Instead of an equalizing effect, resulting in one medium value, an overlapping in the data can be observed, which leads to the conclusion that both solvents affect the pore sizes according to their nature. Compared to the results obtained for samples suspended in only acetone or ethanol (Figs. 2(a and b)), in the histogram of sample Cell ${ }^{\text {ac-et}} \_$S, suspended in the mixture of these solvents, smaller pore sizes $(1100-1300 \mathrm{~nm})$ are also present. The possible explanation for the appearance of smaller pore sizes in this sample is that ethanol can break aggregates up due to its ability to penetrate into them, according to the swelling properties of cellulose in ethanol. The grouping of smaller aggregates results in smaller pores. Another possibility is that the smaller aggregates (resulting from the effects of ethanol) can fit inside the pores formed by bigger stacks (formed due to the effects of acetone) and therefore the relative pore size decreases.

In the histograms of the W-series (Fig. 3) two main groups of columns can be seen for all three samples $\left(\right.$ Cell ${ }^{\text {ac }} \_$W (a), Cell ${ }_{-}^{\text {et }}$ W (b) and Cell $\left.{ }^{\text {ac-et }} \_W(c)\right)$. The first one is in the 10-100 nm interval, while the second one is in the range of $100-1000 \mathrm{~nm}$. For Cellet ${ }^{\mathrm{e}} \mathrm{W}$ the characteristic pore size (the medium of the pore size range with the 

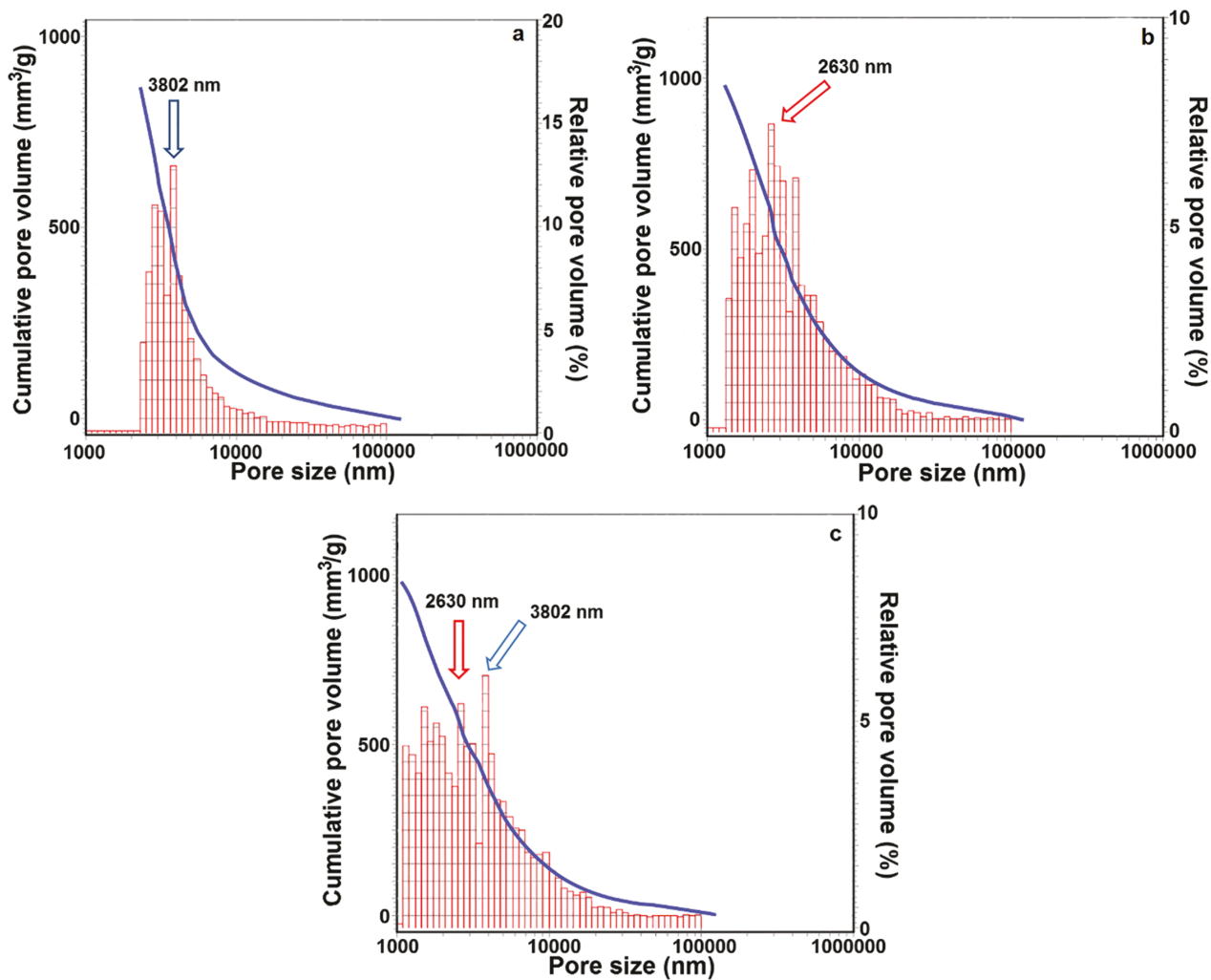

Figure 2. Cumulative (left $y$ axis, blue line) and relative (right $y$ axis, red columns) pore volume versus pore size histograms of Cell ${ }^{\text {ac }} \_S$ (a), Cell ${ }^{\text {et }} \mathrm{S}$ (b) and Cellac-et $\_$S (c).

biggest value in the dataset; not shown here) was $316.2 \mathrm{~nm}$ while for Cell $^{\text {ac }}$ _W it was $416.9 \mathrm{~nm}$. The existence of the first group of columns $(10-100 \mathrm{~nm})$ might derive from the phenomenon when the washing agent penetrates into the fibers of the filter cake and-as from all the used solvents cellulose favors interaction with water-it forms aggregates of NFC in the upper layers of the wet film. After the reorganization of fibers resulting from the penetration of the solvent, the smaller pores, which evolved in the deeper layers during the suspension in water, become accessible.

Comparing all the three histograms of the samples from $\mathrm{W}$-series it can be concluded, that the cumulative pore volume in Cell ${ }^{\text {ac-et }}$ W $\mathrm{W}$ is around the average of the value of the smaller characteristic pore sizes in the histograms of Cell ${ }_{-}^{\text {ac }}$ W and Cell ${ }_{-}^{\text {et }}$ W (Fig. 3). This can be explained with aggregate formation: ethanol makes more, but smaller

Table II. Number of hydrogen bonds per one functional group of a substance acting as donor and acceptor, and the total number of possible hydrogen bonds.

\begin{tabular}{lccc}
\hline Substance & $\begin{array}{c}\text { Hydrogen } \\
\text { donor }\end{array}$ & $\begin{array}{c}\text { Hydrogen } \\
\text { acceptor }\end{array}$ & $\begin{array}{c}\text { Total } \\
\text { H-bonds }\end{array}$ \\
\hline Water & 2 & 2 & 4 \\
Cellulose & 1 & 2 & 3 \\
Acetone & 0 & 2 & 2 \\
Ethanol & 1 & 2 & 3 \\
\hline
\end{tabular}

aggregates, thus in deeper layers these aggregates still have space to form and reveal smaller, earlier inaccessible pores. Acetone, on the other hand, makes bigger aggregates which need bigger space and therefore only a smaller amount of these bigger aggregates can be found in deeper layers. However, when these two solvents are both present, the ethanol-cellulose interactions can loosen up the withholding strength between the fibers thus they become more mobile. This process results in the disclosure of more small pores than in $\mathrm{Cell}^{\mathrm{ac}}{ }_{-} \mathrm{W}$ but, because the concentration of ethanol is lower compared to the case when only pure ethanol was applied as suspending agent, the revealing of smaller pores shall be less intensive, as well.

Even more interesting is how the pore diameters change in higher ranges in the case of Cell ${ }^{\text {ac-et }}$-W, compared to the pore size distribution in Cell ${ }_{-}^{\text {ac }}$ W and $\mathrm{Cell}^{\mathrm{et}}{ }_{-} \mathrm{W}$. When the MIP data are carefully evaluated, similar to the Sseries, there is not a characteristic pore size present in the histogram of Cell ${ }^{\text {ac-et }}$ W $W$ that could be considered as the average value of the characteristic pore sizes of Cell ${ }^{\text {ac }}$-W $(416.9 \mathrm{~nm})$ and Cell ${ }^{\text {et }}$-W $(316.2 \mathrm{~nm})$, but there is a wider interval of the most abundant pore sizes, or in other words: the histogram of Cellac-et $-\mathrm{W}$ is more spread. This wider interval actually occurs due to the overlapping of two groups of histograms, which are visualized in Figure 3(c): one of these groups has the highest value in the pore size interval with the medium of $316.2 \mathrm{~nm}$, and the maximum 

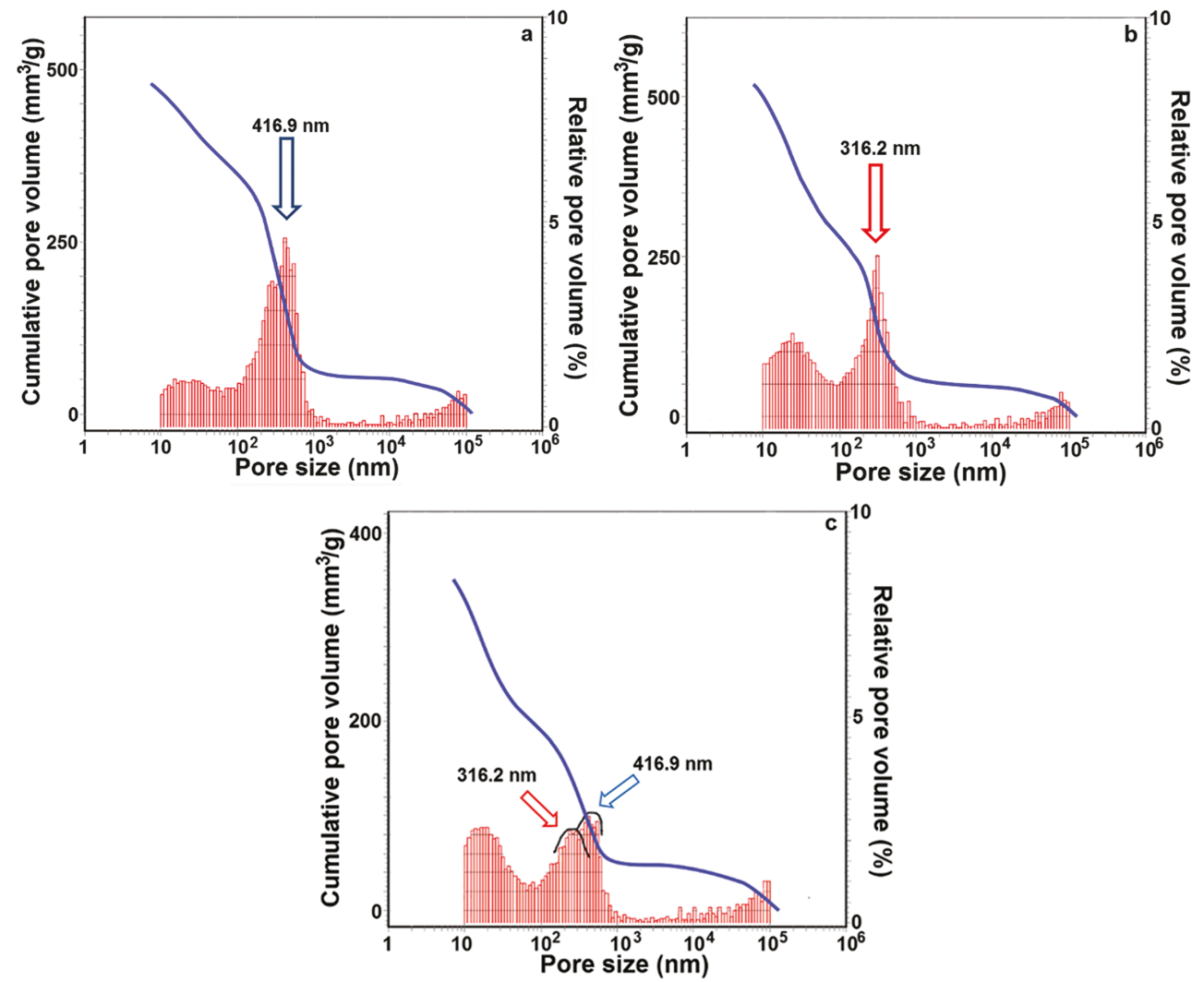

Figure 3. Cumulative (left $y$ axis, blue line) and relative (right $y$ axis, red columns) pore volume versus pore size histograms of Cell ${ }^{\text {ac }}$ W (a), Cell ${ }_{-}^{\text {et }}$ W (b) and Cell ${ }^{\text {ac-et }} \_$W (c). Black lines are approximate fitted curves.

pore size range with value of the other one is in the pore size range with
the medium of $416.9 \mathrm{~nm}$. These media correspond to the characteristic pore sizes of Cell ${ }_{-}^{\text {et }}$ W and Cell ${ }_{-}^{\text {ac }}$ W. However, the relative numerical pore volume values (Table III; extracted from the total dataset, not shown here) of both characteristic pore sizes have decreased radically in the histogram of Cell ${ }^{\text {ac-et }}$-W. If the data in Table III are examined, it is observable that $\mathrm{Cell}^{\mathrm{ac}}{ }_{-} \mathrm{W}$ has a higher relative numerical pore volume value of the pore size range with the medium of $316.2 \mathrm{~nm}$ than Cell ${ }^{\text {ac-et }}$-W, and this phenomenon is also present when the relative numerical pore volume value for the pore size range with the medium of $416.9 \mathrm{~nm}$ is evaluated in the data set of Cellet ${ }^{\mathrm{e}} \mathrm{W}$, compared to the results of Cell ${ }^{\text {ac-et }}$-W.

The radical decrease of relative numerical pore volume values in Cell ${ }^{\text {ac-et }}$ W, suggest a 'quenching' effect of the two solvents, meaning that in the case of the mixture of solvents the resulting sample is less affected by both of

Table III. Relative numerical pore volume values of columns representing characteristic pore sizes for the different solvents in the $\mathrm{W}$-series.

\begin{tabular}{|c|c|c|}
\hline Sample & Medium: $316.2 \mathrm{~nm}$ & Medium: $416.9 \mathrm{~nm}$ \\
\hline Cell $^{\mathrm{ac}} \_\mathrm{W}$ & $16.31 \mathrm{~mm}^{3} / \mathrm{g}$ & $22.13 \mathrm{~mm}^{3} / \mathrm{g}$ \\
\hline Cell $^{\text {et }} \_W$ & $21.83 \mathrm{~mm}^{3} / \mathrm{g}$ & $12.10 \mathrm{~mm}^{3} / \mathrm{g}$ \\
\hline Cell ${ }^{\text {ac-et }} \_W$ & $7.15 \mathrm{~mm}^{3} / \mathrm{g}$ & $9.13 \mathrm{~mm}^{3} / \mathrm{g}$ \\
\hline
\end{tabular}

them, than if the solvents would be present separately. To obtain the 'quenching' ratios (QR) from experimental data, that represent how much the relative numerical pore volumes of the equivalent characteristic pore size columns decreased in Cell ${ }^{\text {ac-et }}$ W compared to those of Cell ${ }^{\mathrm{ac}} \_\mathrm{W}$ and $\mathrm{Cell}^{\mathrm{et}} \_\mathrm{W}$, the relative numerical pore volume values of Cell ${ }^{\text {ac-et }}$-W were normalized to the characteristic pore sizes of Cell ${ }^{\mathrm{ac}}{ }_{-} \mathrm{W}$ and $\mathrm{Cell}^{\mathrm{et}}{ }_{-} \mathrm{W}$, respectively. Thus, the quenching ratios were counted from data shown in Table III as follows:

$$
\begin{aligned}
& \mathrm{QR}_{316.2 \mathrm{~nm}}=\frac{7.15 \mathrm{~mm}^{3}}{21.83 \mathrm{~mm}^{3}} \approx 0.33 \\
& \mathrm{QR}_{416.9 \mathrm{~nm}}=\frac{9.13 \mathrm{~mm}^{3}}{22.13 \mathrm{~mm}^{3}} \approx 0.41
\end{aligned}
$$

As it can be extracted from (1) and (2), the level of 'quenching' is not equal in the cases of two characteristic pore sizes. The strongest possible interaction between cellulose, acetone and ethanol is hydrogen bonding and it also plays a key role in the formation of cellulose nanopapers. As enhanced porosity was obtained after filter cake organization, which is mostly based on hydrogen bonding, it was supposed that the hydrogen bonding ability of the solvents compared to each other must be a crucial point. To prove the theory a simplified mathematical model based on probability computation was proposed. Extracting data from Table II the number of possible distinguished

J. Nanosci. Nanotechnol. 18, 3000-3005, 2018 
hydrogen bonds is 2 between cellulose and acetone (CA), 4 between cellulose and ethanol (CE), 4 between ethanol molecules (EE) and 2 between acetone and ethanol (AE). The case, when the hydroxyl group of cellulose does not bind with any of the solvent molecules is also counted as one possible event (NB). As the 'quenching' is not equal for the different solvents, two probability computations are evaluated-one based on acetone and another one based on ethanol:

$$
\begin{gathered}
P(\mathrm{CA})=\frac{\mathrm{CA}}{\mathrm{CA}+\mathrm{AE}+\mathrm{NB}}=\frac{2}{5}=0.40 \\
P(\mathrm{CE})=\frac{\mathrm{CE}}{\mathrm{CE}+\mathrm{AE}+\mathrm{EE}+\mathrm{NB}}=\frac{4}{11} \approx 0.36
\end{gathered}
$$

These equations can only be interpreted in an idealized system, applying the following considerations:

(i) The solvent distribution is completely homogeneous,

(ii) Every solvent molecule has the same probability to form the possible hydrogen bonds, and

(iii) All the hydrogen bonds are considered to have the same strength.

It can be stated, that the data counted from experimental values $(0.33$ (1) and 0.41 (2) for the pore size ranges with the medium of $316.2 \mathrm{~nm}$ and $416.9 \mathrm{~nm}$, respectively) strongly correlate with the theoretical data $(0.36$ (4) and 0.40 (3) for the pore size ranges with the medium of $316.2 \mathrm{~nm}$ and $416.9 \mathrm{~nm}$, respectively) thus the proposed theory is considered to be proven.

\section{CONCLUSION}

In this study the pore sizes in cellulose nanopapersprepared from NFC suspension-were controlled by solvent exchanging. The aim was not only to refine a process which allows pore size control in cellulose nanopapers, but also to have a better understanding of the ongoing processes on a molecular level, that significantly facilitates the possible future applications.

The swelling properties of cellulose in different solvents derive from their ability of forming hydrogen bonds and therefore highly influence the pore sizes. To support this statement and understand the pore size control mechanism on a molecular level, a simplified mathematical model using probability computations was applied based on experimental data extracted from SEM imaging and MIP measurements. In the case of the W series, a socalled 'quenching effect' has been proposed to explain the observed results. This paper thus proposes a simple, cheap and very fast way to control porosity and pore sizes, which plays a key role in the wide-spectrum application of cellulose nanopapers. High-level accuracy cannot be expected from this method, but in this study efficiency and simplicity possess prior importance, which are crucial in the further use and application of the fabricated structures. However, this mathematical model was sufficiently suitable for strengthening our hypotheses about the system and the ongoing processes in a fast and representative way. These results eventually lead us to the application of nanocellulose as a filter base.

Acknowledgments: We would like to thank the Laboratory of Applied Wood Material (EMPA) for providing the prepared NFC gel. Zoltan Nemeth is thankful for the financial support from the Sciex Programme (project No. 14.119).

\section{References and Notes}

1. D. Klemm, B. Heublein, H. P. Fink, and A. Bohn, Angew Chem. Int. Ed. Engl. 44, 3358 (2005).

2. M. A. S. A. Samir, F. Alloin, and A. Dufresne, Biomacromolecules 6, 612 (2005)

3. S. Ouajai and R. A. Shanks, Compos. Sci. Technol. 69, 2119 (2009).

4. H. Wang, X. Zhang, Z. Jiang, W. Li, and Y. Yu, Industrial Crops and Products 71, 80 (2015).

5. W. H. W. Chen and F. K. Cameron, Ind. Eng. Chem. 34, 224 (1942).

6. A. S. F. Santos, M. A. Pereira-da-Silva, J. E. Oliveira, L. H. C. Mattoso, and E.7S. Medeiros, J. Nanosci. Nanotechnol. 16, 6535 (2016).

7. M. Guinnaraes, V. R. Botaro, K. M. Novack, W. P. F. Neto, L. M Mendes, and G. H. D. Tonoli, J. Nanosci. Nanotechnol. 15, 6751 (2015).

8. Y. Habibi, L. A. Lucia, and O. J. Rojas, Chem. Rev. 110, 3479 (2010).

9. L. N. Fu, J. Zhang, and G. Yang, Carbohydr. Polym. 92, 1432 (2013)

10. S. C. M. Fernandes, C. S. R. Freire, A. J. D. Silvestre, C. P. Neto, A. Gandini, L. A. Berglund, and L. Salmén, Carbohydr. Polym. 81, 394 (2010).

11. N. Lavoine, I. Desloges, A. Dufresne, and J. Bras, Carbohydr. Polym. 90, 735 (2012).

12. C. Salas, T. Nypelö, C. Rodriguez-Abreu, C. Carrillo, and O. J. Rojas, Current Opinion in Colloid and Interface Science 19, 383 (2014).

13. S. R. P. Shariati, S. Moeinzadeh, and E. Jabbari, J. Nanosci. Nanotechnol. 16, 8966 (2016).

14. H. Sehaqui, N. E. Mushi, S. Morimune, M. Salajkova, T. Nishino, and L. A. Berglund, Acs Applied Materials and Interfaces 4, 1043 (2012).

15. M. Henriksson, L. A. Berglund, P. Isaksson, T. Lindstrom, and T. Nishino, Biomacromolecules 9, 1579 (2008).

16. H. Sehaqui, Q. Zhou, O. Ikkala, and L. A. Berglund, Biomacromolecules 12, 3638 (2011).

17. Y. Boluk, Cellulose 12, 577 (2005).

18. K. Missoum, M. Belgacem, and J. Bras, Materials 6, 1745 (2013). 